\title{
Frobenius-Euler polynomials and umbral calculus in the $p$-adic case
}

\section{Dae San Kim ${ }^{1}$, Taekyun Kim²*, Sang-Hun Lee ${ }^{3}$ and Seog-Hoon Rim ${ }^{4}$}

"Correspondence: tkkim@kw.ac.kr ${ }^{2}$ Department of Mathematics, Kwangwoon University, Seoul, 139-701, Republic of Korea

Full list of author information is available at the end of the article

\begin{abstract}
In this paper, we study some $p$-adic Frobenius-Euler measure related to umbral calculus in the $p$-adic case. Finally, we derive some identities of Frobenius-Euler polynomials from our study.
\end{abstract}

MSC: 05A10;05A19

Keywords: Frobenius-Euler polynomials; umbral calculus; $p$-adic integral

\section{Introduction}

Let $p$ be a fixed prime number. Throughout this paper $\mathbb{Z}_{p}, \mathbb{Q}_{p}$ and $\mathbb{C}_{p}$ will denote the ring of $p$-adic integers, the field of $p$-adic rational numbers and the completion of algebraic closure of $\mathbb{Q}_{p}$, respectively.

$$
\begin{aligned}
& \text { For } f \in \mathbb{N} \text { with }(f, p)=1 \text {, let } \\
& \begin{aligned}
X & =\lim _{\overleftarrow{N}} \mathbb{Z} / f p^{N} \mathbb{Z} ; \\
a & +f p^{N} \mathbb{Z}_{p}=\left\{x \in X \mid x \equiv a\left(\bmod f p^{N}\right)\right\}, \quad 0 \leq a \leq f p^{N}-1, \\
X^{*} & =\bigcup_{0<a<f p,(a, p)=1}\left(a+f p^{N} \mathbb{Z}_{p}\right), \quad N \in \mathbb{N}(\text { see }[1-7]) .
\end{aligned}
\end{aligned}
$$

Note that the natural map $\mathbb{Z} / f p^{N} \mathbb{Z} \rightarrow \mathbb{Z} / p^{N} \mathbb{Z}$ induces

$$
\pi: X \rightarrow \mathbb{Z}_{p}
$$

If $g$ is a function on $\mathbb{Z}_{p}$, we denote by the same $g$ the function $g \circ \pi$ on $X$. Namely, we can consider $g$ as a function on $X$.

For $k \geq 0$ and $\lambda \in \mathbb{C}_{p}$ with $|1-\lambda|_{p}>1$, the Frobenius-Euler measure on $X$ is defined by

$$
\mu_{\lambda}\left(x+f p^{N} \mathbb{Z}_{p}\right)=\frac{\lambda^{f p^{N}-x}}{1-\lambda p^{N}} \quad(\text { see }[5,8]),
$$

where the $p$-adic absolute value on $\mathbb{C}_{p}$ is normalized by $|p|_{p}=\frac{1}{p}$.

As is well known, the Frobenius-Euler polynomials are defined by the generating function to be

$$
\left(\frac{1-\lambda}{e^{t}-\lambda}\right) e^{x t}=e^{H(x \mid \lambda) t}=\sum_{n=0}^{\infty} H_{n}(x \mid \lambda) \frac{t^{n}}{n !} \quad(\text { see }[5,7,9]),
$$

\section{Springer}

(c) 2012 Kim et al: licensee Springer. This is an Open Access article distributed under the terms of the Creative Commons Attribution License (http://creativecommons.org/licenses/by/2.0), which permits unrestricted use, distribution, and reproduction in any medium, provided the original work is properly cited. 
with the usual convention about replacing $H^{n}(x \mid \lambda)$ by $H_{n}(x \mid \lambda)$. In the special case, $x=0$, $H_{n}(0 \mid \lambda)=H_{n}(\lambda)$ are called the $n$th Frobenius-Euler numbers

$$
H_{n}(x \mid \lambda)=(H(\lambda)+x)^{n}=\sum_{l=0}^{\infty}\left(\begin{array}{l}
n \\
l
\end{array}\right) H_{l}(\lambda) x^{n-l} \quad(\text { see }[6,9,10]) .
$$

Thus, by (1.2) and (1.3), we easily get

$$
(H(\lambda)+1)^{n}-\lambda H_{n}(\lambda)=(1-\lambda) \delta_{0, n} \quad(\text { see }[1-19]),
$$

where $\delta_{n, k}$ is the Kronecker symbol.

For $r \in \mathbb{N}$, the Frobenius-Euler polynomials of order $r$ are defined by the generating function

$$
\begin{aligned}
\left(\frac{1-\lambda}{e^{t}-\lambda}\right)^{r} e^{x t} & =\underbrace{\left(\frac{1-\lambda}{e^{t}-\lambda}\right) \times \cdots \times\left(\frac{1-\lambda}{e^{t}-\lambda}\right)}_{r \text {-times }} e^{x t} \\
& =\sum_{n=0}^{\infty} H_{n}^{(r)}(x \mid \lambda) \frac{t^{n}}{n !} \quad(\text { see }[5,9]) .
\end{aligned}
$$

In the special case, $x=0, H_{n}^{(r)}(0 \mid \lambda)=H_{n}^{(r)}(\lambda)$ are called the $n$th Frobenius-Euler numbers of order $r$. The $n$th Frobenius-Euler polynomials can be represented by (1.1) as follows:

$$
\begin{aligned}
\frac{\lambda H_{n}(x \mid \lambda)}{1-\lambda} & =\int_{X}(x+y)^{n} d \mu_{\lambda}(y)=\int_{\mathbb{Z}_{p}}(x+y)^{n} d \mu_{\lambda}(y) \\
& \left.=\lim _{N \rightarrow \infty} \frac{1}{1-\lambda p^{N}} \sum_{y=0}^{p^{N}-1}(x+y)^{n} \lambda^{p^{N}-y} \quad \text { (see }[6,7]\right) .
\end{aligned}
$$

Let $\mathcal{F}$ be the set of all formal power series in the variable $t$ over $\mathbb{C}_{p}$ with

$$
\mathcal{F}=\left\{f(t)=\sum_{k=0}^{\infty} \frac{a_{k}}{k !} t^{k} \mid a_{k} \in \mathbb{C}_{p}\right\} .
$$

Let $\mathbb{P}=\mathbb{C}_{p}[x]$ and $\mathbb{P}^{*}$ denote the vector space of all linear functionals on $\mathbb{P}$.

The formal power series

$$
f(t)=\sum_{k=0}^{\infty} \frac{a_{k}}{k !} t^{k} \in \mathcal{F} \quad(\text { see }[11,15])
$$

defines a linear functional on $\mathbb{P}$ by setting

$$
\left\langle f(t) \mid x^{n}\right\rangle=a_{n} \quad \text { for all } n \geq 0 .
$$

From (1.8) and (1.9), we have

$$
\left\langle t^{n} \mid x^{n}\right\rangle=n ! \delta_{n, k} \quad(n, k \geq 0)
$$


Here, $\mathcal{F}$ denotes both the algebra of formal power series in $t$ and the vector space of all linear functionals on $\mathbb{P}$, and so an element $f(t)$ of $\mathcal{F}$ will be thought of as both a formal power series and a linear functional (see $[11,15])$. We will call $\mathcal{F}$ the umbral algebra. The umbral calculus is the study of umbral algebra (see $[11,15])$.

The order $o(f(t))$ of power series $f(t)(\neq 0)$ is the smallest integer $k$ for which $a_{k}$ does not vanish (see [11, 15]). A series $f(t)$ for which $o(f(t))=1$ is called a delta series. If a series $f(t)$ has $o(f(t))=0$, then $f(t)$ is called an invertible series (see [11, 15]). Let $f(t), g(t) \in \mathcal{F}$. Then we easily see that $\langle f(t) g(t) \mid p(x)\rangle=\langle f(t) \mid g(t) p(x)\rangle=\langle g(t) \mid f(t) p(x)\rangle$. From (1.10), we note that

$$
\begin{aligned}
& \left\langle e^{y t} \mid x^{n}\right\rangle=y^{n}, \quad\left\langle e^{y t} \mid p(x)\right\rangle=p(y), \\
& f(t)=\sum_{k=0}^{\infty} \frac{\left\langle f(t) \mid x^{k}\right\rangle}{k !} t^{k}, \quad f(t) \in \mathcal{F},
\end{aligned}
$$

and

$$
p(x)=\sum_{k=0}^{\infty} \frac{\left\langle t^{k} \mid p(x)\right\rangle}{k !} x^{k}, \quad p(x) \in \mathbb{P}(\text { see }[15])
$$

For $f_{1}(t), f_{2}(t), \ldots, f_{m}(t) \in \mathcal{F}$, we have

$$
\left\langle f_{1}(t) f_{2}(t) \cdots f_{m}(t) \mid x^{n}\right\rangle=\sum_{i_{1}+\cdots+i_{m}=n}\left(\begin{array}{c}
n \\
i_{1}, \ldots, i_{m}
\end{array}\right)\left\langle f_{1}(t) \mid x^{i_{1}}\right\rangle \cdots\left\langle f_{m}(t) \mid x^{i_{m}}\right\rangle
$$

By (1.13), we get

$$
p^{(k)}(x)=\frac{d^{k} p(x)}{d x^{k}}=\sum_{l=k}^{n}\left\langle t^{l} \mid p(x)\right\rangle\left(\begin{array}{l}
l \\
k
\end{array}\right) \frac{k !}{l !} x^{l-k}
$$

and

$$
p^{(k)}(0)=\left\langle t^{k} \mid p(x)\right\rangle=\left\langle 1 \mid p^{(k)}(x)\right\rangle
$$

Thus, by (1.15), we get

$$
t^{k} p(x)=p^{(k)}(x)=\frac{d^{k} p(x)}{d x^{k}} \quad(\text { see }[11,15])
$$

By (1.16), we easily see that

$$
e^{y t} p(x)=p(x+y) \quad(\text { see }[15])
$$

Let $S_{n}(x)$ denote a polynomial of degree $n$. Suppose that $f(t), g(t) \in \mathcal{F}$ with $o(f(t))=1$ and $o(g(t))=0$. Then there exists a unique sequence $S_{n}(x)$ of polynomials satisfying $\left\langle g(t) f(t)^{k} \mid S_{n}(x)\right\rangle=n ! \delta_{n, k}$ for all $n, k \geq 0$. The sequence $S_{n}(x)$ is called the Sheffer sequence for $(g(t), f(t))$, which is denoted by $S_{n}(x) \sim(g(t), f(t))$. If $S_{n}(x) \sim(g(t), t)$, then $S_{n}(x)$ is called the Appell sequence for $g(t)$ (see [15]). 
For $p(x) \in \mathbb{P}$, we have

$$
\begin{aligned}
& \langle f(t) \mid x p(x)\rangle=\left\langle\partial_{t} f(t) \mid p(x)\right\rangle=\left\langle f^{\prime}(t) \mid p(x)\right\rangle, \\
& \left\langle e^{y t}-1 \mid p(x)\right\rangle=p(y)-p(0) .
\end{aligned}
$$

If $S_{n}(x) \sim(g(t), f(t))$, then we have

$$
\begin{aligned}
& h(t)=\sum_{k=0}^{\infty} \frac{\left\langle h(t) \mid S_{k}(x)\right\rangle}{k !} g(t) f(t)^{k}, \quad h(t) \in \mathcal{F}, \\
& p(x)=\sum_{k=0}^{\infty} \frac{\left\langle g(t) f(t)^{k} \mid p(x)\right\rangle}{k !} S_{k}(x), \quad p(x) \in \mathbb{P}, \\
& f(t) S_{n}(x)=n S_{n-1}(x),
\end{aligned}
$$

and

$$
\frac{1}{g(\bar{f}(t))} e^{\overline{y f}(t)}=\sum_{k=0}^{\infty} \frac{S_{k}(y)}{k !} t^{k} \quad \text { for all } y \in \mathbb{C}_{p}
$$

where $\bar{f}(t)$ is compositional inverse of $f(t)$ (see [11, 15]). In [9], Kim and Kim have studied some identities of Frobenius-Euler polynomials arising from umbral calculus. In this paper, we study some $p$-adic Frobenius-Euler integral on $\mathbb{Z}_{p}$ related to umbral calculus in the $p$-adic case. Finally, we derive some new and interesting identities of Frobenius-Euler polynomials from our study.

\section{Frobenius-Euler polynomials associated with umbral calculus}

Let

$$
g(t ; \lambda)=\frac{e^{t}-\lambda}{1-\lambda} \in \mathcal{F}
$$

Then we see that $g(t ; \lambda)$ is an invertible series. From (1.2), we have

$$
\sum_{k=0}^{\infty} H_{k}(x \mid \lambda) \frac{t^{k}}{k !}=\frac{1}{g(t ; \lambda)} e^{x t}
$$

Hence, by (2.2), we get

$$
\left(\frac{1-\lambda}{e^{t}-\lambda}\right) x^{n}=\frac{1}{g(t ; \lambda)} x^{n}=H_{n}(x \mid \lambda)
$$

By (2.2) and (2.3), we get

$$
H_{n}(x \mid \lambda) \sim(g(t ; \lambda), t) .
$$

From (1.6), we have

$$
\int_{\mathbb{Z}_{p}} e^{(x+y) t} d \mu_{\lambda}(y)=\frac{\lambda}{e^{t}-\lambda} e^{x t},
$$


and

$$
\int_{\mathbb{Z}_{p}} e^{(x+y+1) t} d \mu_{\lambda}(y)-\lambda \int_{\mathbb{Z}_{p}} e^{(x+y) t} d \mu_{\lambda}(y)=\lambda e^{x t}
$$

By (2.5), we get

$$
\int_{\mathbb{Z}_{p}}(x+y+1)^{n} d \mu_{\lambda}(y)-\lambda \int_{\mathbb{Z}_{p}}(x+y)^{n} d \mu_{\lambda}(y)=\lambda x^{n} .
$$

From (1.6) and (2.6), we have

$$
\frac{\lambda}{1-\lambda} H_{n}(x+1 \mid \lambda)-\frac{\lambda^{2}}{1-\lambda} H_{n}(x \mid \lambda)=\lambda x^{n} .
$$

From (2.2), we can easily derive

$$
H_{n+1}(x \mid \lambda)=\left(x-\frac{g^{\prime}(t ; \lambda)}{g(t ; \lambda)}\right) H_{n}(x \mid \lambda) .
$$

By (2.8), we get

$$
g(t ; \lambda) H_{n+1}(x \mid \lambda)=g(t ; \lambda) x H_{n}(x \mid \lambda)-g^{\prime}(t ; \lambda) H_{n}(x \mid \lambda) .
$$

Thus, from (2.9), we have

$$
\left(e^{t}-\lambda\right) H_{n+1}(x \mid \lambda)=\left(e^{t}-\lambda\right) x H_{n}(x \mid \lambda)-e^{t} H_{n}(x \mid \lambda) .
$$

By (2.10), we get

$$
H_{n+1}(x+1 \mid \lambda)-\lambda H_{n+1}(x \mid \lambda)=x\left(H_{n}(x+1 \mid \lambda)-\lambda H_{n}(x \mid \lambda)\right) .
$$

From (2.11), we note that

$$
\begin{aligned}
H_{n}(x+1 \mid \lambda)-\lambda H_{n}(x \mid \lambda) & =x\left(H_{n-1}(x+1 \mid \lambda)-\lambda H_{n-1}(x \mid \lambda)\right) \\
& =x^{2}\left(H_{n-2}(x+1 \mid \lambda)-\lambda H_{n-2}(x \mid \lambda)\right)=\cdots \\
& =x^{n}\left(H_{0}(x+1 \mid \lambda)-\lambda H_{0}(x \mid \lambda)\right)=x^{n}(1-\lambda) .
\end{aligned}
$$

Let us consider the linear functional $f(t)$ such that

$$
\langle f(t) \mid p(x)\rangle=\int_{\mathbb{Z}_{p}} p(u) d \mu_{\lambda}(u)
$$

for all polynomials $p(x)$ can be determined from (1.12) to be

$$
f(t)=\sum_{k=0}^{\infty} \frac{\left\langle f(t) \mid x^{k}\right\rangle}{k !} t^{k}=\sum_{k=0}^{\infty} \int_{\mathbb{Z}_{p}} u^{k} d \mu_{\lambda}(u) \frac{t^{k}}{k !}=\int_{\mathbb{Z}_{p}} e^{u t} d \mu_{\lambda}(u) .
$$


By (2.4) and (2.14), we get

$$
f(t)=\int_{\mathbb{Z}_{p}} e^{u t} d \mu_{\lambda}(u)=\frac{\lambda}{e^{t}-\lambda} .
$$

Therefore, by (2.15), we obtain the following theorem.

Theorem 2.1 For $p(x) \in \mathbb{P}$, we have

$$
\left\langle\frac{\lambda}{e^{t}-\lambda} \mid p(x)\right\rangle=\int_{\mathbb{Z}_{p}} p(u) d \mu_{\lambda}(u) .
$$

In particular,

$$
\frac{\lambda}{1-\lambda} H_{n}(\lambda)=\left\langle\int_{\mathbb{Z}_{p}} e^{y t} d \mu_{\lambda}(y) \mid x^{n}\right\rangle
$$

From (1.6), we have

$$
\sum_{n=0}^{\infty} \int_{\mathbb{Z}_{p}}(x+y)^{n} d \mu_{\lambda}(y) \frac{t^{n}}{n !}=\int_{\mathbb{Z}_{p}} e^{(x+y) t} d \mu_{\lambda}(y)=\sum_{n=0}^{\infty} \int_{\mathbb{Z}_{p}} e^{y t} d \mu_{\lambda}(y) x^{n} \frac{t^{n}}{n !}
$$

By (1.6), (2.4) and (2.16), we get

$$
\frac{\lambda}{1-\lambda} H_{n}(x \mid \lambda)=\int_{\mathbb{Z}_{p}} e^{y t} d \mu_{\lambda}(y) x^{n}=\frac{\lambda}{e^{t}-\lambda} x^{n}, \quad \text { for } n \geq 0 .
$$

Therefore, by (2.17), we obtain the following theorem.

Theorem 2.2 For $p(x) \in \mathbb{P}$, we have

$$
\int_{\mathbb{Z}_{p}} p(x+y) d \mu_{\lambda}(y)=\int_{\mathbb{Z}_{p}} e^{y t} d \mu_{\lambda}(y) p(x)=\frac{\lambda}{e^{t}-\lambda} p(x) .
$$

In particular,

$$
\frac{\lambda}{1-\lambda} H_{n}(x \mid \lambda)=\int_{\mathbb{Z}_{p}} e^{y t} d \mu_{\lambda}(y) x^{n}=\frac{\lambda}{e^{t}-\lambda} x^{n} \quad(n \geq 0) .
$$

By (1.6) and (2.16), we get

$$
\frac{\lambda}{1-\lambda} H_{n}(x \mid \lambda) \sim\left(\frac{e^{t}-\lambda}{\lambda}, t\right) .
$$

From Appell identity and (2.18), we can derive the following identities:

$$
H_{n}(x+y \mid \lambda)=\sum_{k=0}^{n}\left(\begin{array}{l}
n \\
k
\end{array}\right) H_{k}(x \mid \lambda) y^{n-k} .
$$


Let

$$
g^{r}(t ; \lambda)=\left(\frac{e^{t}-\lambda}{\lambda}\right)^{r}=\underbrace{\left(\frac{e^{t}-\lambda}{\lambda}\right) \times \cdots \times\left(\frac{e^{t}-\lambda}{\lambda}\right)}_{r \text {-times }} \in \mathcal{F} .
$$

Then $g^{r}(t ; \lambda)$ is an invertible functional in $\mathcal{F}$. By (1.5) and (2.20), we get

$$
\frac{1}{g^{r}(t ; \lambda)} e^{x t}=\frac{\lambda^{r}}{(1-\lambda)^{r}} \sum_{k=0}^{\infty} H_{n}^{(r)}(x \mid \lambda) \frac{t^{n}}{n !}
$$

Thus, from (2.21), we have

$$
\frac{1}{g^{r}(t ; \lambda)} x^{n}=\left(\frac{\lambda}{1-\lambda}\right)^{r} H_{n}^{(r)}(x \mid \lambda),
$$

and

$$
\left(\frac{\lambda}{1-\lambda}\right)^{r} t H_{n}^{(r)}(x \mid \lambda)=\frac{n}{g^{r}(t ; \lambda)} x^{n-1}=n\left(\frac{\lambda}{1-\lambda}\right)^{r} H_{n-1}^{(r)}(x \mid \lambda) .
$$

By (2.22) and (2.23), we see that

$$
\left(\frac{\lambda}{1-\lambda}\right)^{r} H_{n}^{(r)}(x \mid \lambda) \sim\left(g^{r}(t ; \lambda), t\right) .
$$

From (2.4), we can derive the following identity:

$$
\begin{aligned}
& \underbrace{\int_{\mathbb{Z}_{p}} \cdots \int_{\mathbb{Z}_{p}} e^{\left(x_{1}+x_{2}+\cdots+x_{r}+x\right) t} d \mu_{\lambda}\left(x_{1}\right) \cdots d \mu_{\lambda}\left(x_{r}\right)}_{r \text {-times }} \\
& =\left(\frac{\lambda}{e^{t}-\lambda}\right)^{r} e^{x t}=\left(\frac{\lambda}{1-\lambda}\right)^{r} \sum_{n=0}^{\infty} H_{n}^{(r)}(x \mid \lambda) \frac{t^{n}}{n !} .
\end{aligned}
$$

By (1.10) and (2.25), we get

$$
\begin{aligned}
& \left(\frac{\lambda}{1-\lambda}\right)^{r} H_{n}^{(r)}(x \mid \lambda) \\
& \quad=\langle\underbrace{\int_{\mathbb{Z}_{p}} \cdots \int_{\mathbb{Z}_{p}} e^{\left(x_{1}+x_{2}+\cdots+x_{r}+x\right) t} d \mu_{\lambda}\left(x_{1}\right) \cdots d \mu_{\lambda}\left(x_{r}\right)\left|x^{n}\right\rangle .}_{r \text {-times }}
\end{aligned}
$$

From (1.14), we have

$$
\begin{aligned}
& \langle\underbrace{\int_{\mathbb{Z}_{p}} \cdots \int_{\mathbb{Z}_{p}} e^{\left(x_{1}+x_{2}+\cdots+x_{r}\right) t} d \mu_{\lambda}\left(x_{1}\right) \cdots d \mu_{\lambda}\left(x_{r}\right)\left|x^{n}\right\rangle}_{r \text {-times }} \\
& =\sum_{n=i_{1}+\cdots+i_{r}}\left(\begin{array}{c}
n \\
i_{1}, \ldots, i_{r}
\end{array}\right)\left\langle\int_{\mathbb{Z}_{p}} e^{x_{1} t} d \mu_{\lambda}\left(x_{1}\right) \mid x^{i_{1}}\right\rangle \times \cdots
\end{aligned}
$$




$$
\begin{aligned}
& \times\left\langle\int_{\mathbb{Z}_{p}} e^{x_{r} t} d \mu_{\lambda}\left(x_{r}\right) \mid x^{i_{r}}\right\rangle \\
= & \sum_{n=i_{1}+\cdots+i_{r}}\left(\begin{array}{c}
n \\
i_{1}, \ldots, i_{r}
\end{array}\right)\left(\frac{\lambda}{1-\lambda}\right)^{r} H_{i_{1}}(x \mid \lambda) \cdots H_{i_{r}}(x \mid \lambda) .
\end{aligned}
$$

By (2.26) and (2.27), we get

$$
H_{n}^{(r)}(x \mid \lambda)=\sum_{n=i_{1}+\cdots+i_{r}}\left(\begin{array}{c}
n \\
i_{1}, \ldots, i_{r}
\end{array}\right) H_{i_{1}}(x \mid \lambda) \cdots H_{i_{r}}(x \mid \lambda)
$$

where $\left(\begin{array}{c}n \\ i_{1}, \ldots, i_{r}\end{array}\right)=\frac{n !}{i_{1} ! \cdots i_{r} !}$. From (2.25), we note that

$$
g^{r}(t ; \lambda)=\underbrace{\frac{1}{\int_{\mathbb{Z}_{p}} \cdots \int_{\mathbb{Z}_{p}}} e^{\left(x_{1}+x_{2}+\cdots+x_{r}\right) t} d \mu_{\lambda}\left(x_{1}\right) \cdots d \mu_{\lambda}\left(x_{r}\right)}_{r \text {-times }}=\left(\frac{e^{t}-\lambda}{\lambda}\right)^{r} .
$$

Thus, by (2.28), we get

$$
\begin{aligned}
\frac{1}{g^{r}(t ; \lambda)} e^{x t} & =\int_{\mathbb{Z}_{p}} \cdots \int_{\mathbb{Z}_{p}} e^{\left(x_{1}+x_{2}+\cdots+x_{r}\right) t} d \mu_{\lambda}\left(x_{1}\right) \cdots d \mu_{\lambda}\left(x_{r}\right) e^{x t} \\
& =\int_{\mathbb{Z}_{p}} \cdots \int_{\mathbb{Z}_{p}} e^{\left(x_{1}+x_{2}+\cdots+x_{r}+x\right) t} d \mu_{\lambda}\left(x_{1}\right) \cdots d \mu_{\lambda}\left(x_{r}\right) \\
& =\left(\frac{\lambda}{1-\lambda}\right)^{r} \sum_{n=0}^{\infty} H_{n}^{(r)}(x \mid \lambda) \frac{t^{n}}{n !} .
\end{aligned}
$$

By (2.29), we see that

$$
\begin{aligned}
& \left(\frac{\lambda}{1-\lambda}\right)^{r} H_{n}^{(r)}(x \mid \lambda) \\
& =\underbrace{\int_{\mathbb{Z}_{p}} \cdots \int_{\mathbb{Z}_{p}}\left(x+x_{1}+\cdots+x_{r}\right)^{n} d \mu_{\lambda}\left(x_{1}\right) \cdots d \mu_{\lambda}\left(x_{r}\right)}_{r \text {-times }} \\
& =\underbrace{\int_{\mathbb{Z}_{p}} \cdots \int_{\mathbb{Z}_{p}}}_{r \text {-times }} e^{\left(x_{1}+x_{2}+\cdots+x_{r}\right) t} d \mu_{\lambda}\left(x_{1}\right) \cdots d \mu_{\lambda}\left(x_{r}\right) x^{n} \\
& =\frac{1}{g^{r}(t ; \lambda)} x^{n} .
\end{aligned}
$$

Therefore, by (2.30), we obtain the following theorem.

Theorem 2.3 For $p(x) \in \mathbb{P}$ and $r \in \mathbb{N}$, we have

$$
\underbrace{\int_{\mathbb{Z}_{p}} \cdots \int_{\mathbb{Z}_{p}}}_{r \text {-times }} p\left(x_{1}+x_{2}+\cdots+x_{r}+x\right) d \mu_{\lambda}\left(x_{1}\right) \cdots d \mu_{\lambda}\left(x_{r}\right)=\left(\frac{\lambda}{e^{t}-\lambda}\right)^{r} p(x) .
$$


In particular,

$$
\left(\frac{\lambda}{1-\lambda}\right)^{r} H_{n}^{(r)}(x \mid \lambda)=\int_{\mathbb{Z}_{p}} \cdots \int_{\mathbb{Z}_{p}} e^{\left(x_{1}+x_{2}+\cdots+x_{r}\right) t} d \mu_{\lambda}\left(x_{1}\right) \cdots d \mu_{\lambda}\left(x_{r}\right) x^{n} .
$$

Moreover,

$$
\left(\frac{\lambda}{1-\lambda}\right)^{r} H_{n}^{(r)}(x \mid \lambda) \sim\left(\frac{1}{\int_{\mathbb{Z}_{p}} \cdots \int_{\mathbb{Z}_{p}} e^{\left(x_{1}+x_{2}+\cdots+x_{r}\right) t} d \mu_{\lambda}\left(x_{1}\right) \cdots d \mu_{\lambda}\left(x_{r}\right)}, t\right) .
$$

Let us consider the function $f^{*}(t)$ in $\mathcal{F}$ such that

$$
\left\langle f^{*}(t) \mid p(x)\right\rangle=\int_{\mathbb{Z}_{p}} \cdots \int_{\mathbb{Z}_{p}} p\left(x_{1}+x_{2}+\cdots+x_{r}\right) d \mu_{\lambda}\left(x_{1}\right) \cdots d \mu_{\lambda}\left(x_{r}\right)
$$

for all polynomials $p(x)$ can be determined from (1.12) to be

$$
\begin{aligned}
f^{*}(t) & =\sum_{k=0}^{\infty} \frac{\left\langle f^{*}(t) \mid x^{k}\right\rangle}{k !} t^{k} \\
& =\sum_{k=0}^{\infty} \underbrace{\int_{\mathbb{Z}_{p}} \cdots \int_{\mathbb{Z}_{p}}}_{r \text {-times }}\left(x_{1}+\cdots+x_{r}\right)^{k} d \mu_{\lambda}\left(x_{1}\right) \cdots d \mu_{\lambda}\left(x_{r}\right) \frac{t^{k}}{k !} \\
& =\underbrace{\int_{\mathbb{Z}_{p}} \cdots \int_{\mathbb{Z}_{p}} e^{\left(x_{1}+\cdots+x_{r}\right) t} d \mu_{\lambda}\left(x_{1}\right) \cdots d \mu_{\lambda}\left(x_{r}\right) .}_{r \text {-times }}
\end{aligned}
$$

Therefore, by (2.31) and (2.32), we obtain the following theorem.

Theorem 2.4 For $p(x) \in \mathbb{P}$, we have

$$
\begin{aligned}
& \langle\underbrace{\int_{\mathbb{Z}_{p}} \cdots \int_{\mathbb{Z}_{p}} e^{\left(x_{1}+x_{2}+\cdots+x_{r}\right) t} d \mu_{\lambda}\left(x_{1}\right) \cdots d \mu_{\lambda}\left(x_{r}\right)|p(x)\rangle}_{r \text {-times }} \\
& =\underbrace{\int_{\mathbb{Z}_{p}} \cdots \int_{\mathbb{Z}_{p}} p\left(x_{1}+x_{2}+\cdots+x_{r}\right) d \mu_{\lambda}\left(x_{1}\right) \cdots d \mu_{\lambda}\left(x_{r}\right) .}_{r \text {-times }}
\end{aligned}
$$

In particular,

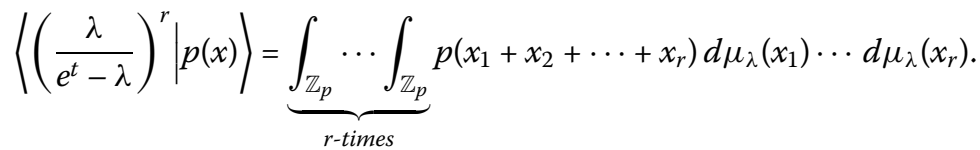

Indeed, the $n$th Frobenius-Euler number of order $r$ is given by

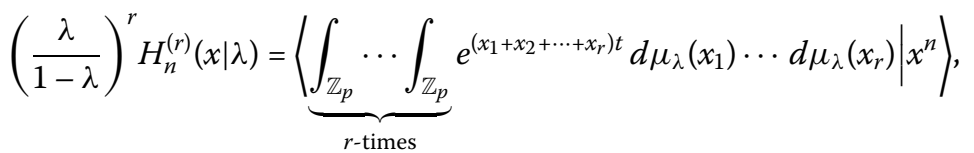

where $n \geq 0$. 
Remark From (1.2) and (1.5), we note that

$$
\begin{aligned}
\frac{d}{d \lambda}\left(\frac{1-\lambda}{e^{t}-\lambda}\right) & =\frac{1-e^{t}}{\left(e^{t}-\lambda\right)^{2}}=\frac{1}{(1-\lambda)}\left(\frac{(1-\lambda)^{2}}{\left(e^{t}-\lambda\right)^{2}}-\frac{1-\lambda}{e^{t}-\lambda}\right) \\
& =\frac{1}{1-\lambda} \sum_{n=0}^{\infty}\left(H_{n}^{(2)}(\lambda)-H_{n}(\lambda)\right) \frac{t^{n}}{n !},
\end{aligned}
$$

and

$$
\begin{aligned}
\frac{d^{2}}{d \lambda^{2}}\left(\frac{1-\lambda}{e^{t}-\lambda}\right) & =2 ! \frac{1-e^{t}}{\left(e^{t}-\lambda\right)^{3}}=\frac{2 !}{(1-\lambda)^{2}}\left(\frac{(1-\lambda)^{3}}{\left(e^{t}-\lambda\right)^{3}}-\frac{(1-\lambda)^{2}}{\left(e^{t}-\lambda\right)^{2}}\right) \\
& =\frac{2 !}{(1-\lambda)^{2}} \sum_{n=0}^{\infty}\left(H_{n}^{(3)}(\lambda)-H_{n}^{(2)}(\lambda)\right) \frac{t^{n}}{n !} .
\end{aligned}
$$

Continuing this process, we obtain the following equation:

$$
\begin{aligned}
\frac{d^{k}}{d \lambda^{k}}\left(\frac{1-\lambda}{e^{t}-\lambda}\right) & =\frac{k !}{(1-\lambda)^{k}}\left(\frac{(1-\lambda)^{k+1}}{\left(e^{t}-\lambda\right)^{k+1}}-\frac{(1-\lambda)^{k}}{\left(e^{t}-\lambda\right)^{k}}\right) \\
& =\frac{k !}{(1-\lambda)^{k}} \sum_{n=0}^{\infty}\left(H_{n}^{(k+1)}(\lambda)-H_{n}^{(k)}(\lambda)\right) \frac{t^{n}}{n !} .
\end{aligned}
$$

By (1.2), (1.5) and (2.35), we get

$$
\frac{d^{k}}{d \lambda^{k}} H_{n}(\lambda)=\frac{k !}{(1-\lambda)^{k}}\left(H_{n}^{(k+1)}(\lambda)-H_{n}^{(k)}(\lambda)\right)
$$

where $k$ is a positive integer.

\section{Competing interests}

The authors declare that they have no competing interests.

\section{Authors' contributions}

All authors contributed equally to the manuscript and typed, read, and approved the final manuscript.

\section{Author details}

1Department of Mathematics, Sogang University, Seoul, 121-741, Republic of Korea. ${ }^{2}$ Department of Mathematics, Kwangwoon University, Seoul, 139-701, Republic of Korea. ${ }^{3}$ Division of General Education, Kwangwoon University, Seoul, 139-701, Republic of Korea. ${ }^{4}$ Department of Mathematics Education, Kyungpook National University, Taegu, 702-701, Republic of Korea.

\section{Acknowledgements}

This research was supported by the Basic Science Research Program through the National Research Foundation of Korea (NRF) funded by the Ministry of Education, Science and Technology 2012R1A1A2003786.

Received: 21 November 2012 Accepted: 6 December 2012 Published: 20 December 2012

\section{References}

1. Araci, S, Acikgoz, M: A note on the Frobenius-Euler numbers and polynomials associated with Bernstein polynomials. Adv. Stud. Contemp. Math. 22, 399-406 (2012)

2. Can, M, Cenkci, M, Kurt, V, Simsek, Y: Twisted Dedekind type sums associated with Barnes' type multiple Frobenius-Euler I-functions. Adv. Stud. Contemp. Math. 18, 135-160 (2009)

3. Carlitz, L: The product of two Eulerian polynomials. Math. Mag. 368, 37-41 (1963)

4. Dere, R, Simsek, Y: Applications of umbral algebra to some special polynomials. Adv. Stud. Contemp. Math. 22, 433-438 (2012)

5. Kim, T: On explicit formulas of p-adic q-L-functions. Kyushu J. Math. 48, 73-86 (1994) 
6. Kim, T, Choi, J: A note on the product of Frobenius-Euler polynomials arising from the $p$-adic integral on $\mathbb{Z}_{p}$. Adv. Stud. Contemp. Math. 22, 215-223 (2012)

7. Kim, T, Lee, B: Some identities of the Frobenius-Euler polynomials. Abstr. Appl. Anal. 2009, Article ID 639439 (2009)

8. Shiratani, K, Yamamoto, S: On a $p$-adic interpolation function for the Euler numbers and its derivatives. Mem. Fac. Sci., Kyushu Univ., Ser. A 39, 113-125 (1985)

9. Kim, DS, Kim, T: Some identities of Frobenius-Euler polynomials arising from umbral calculus. Adv. Differ. Equ. 2012 196 (2012). doi:10.1186/1687-1847-2012-196

10. Ryoo, CS: A note on the Frobenius-Euler polynomials. Proc. Jangjeon Math. Soc. 14, 495-501 (2014)

11. Kim, T: Identities involving Frobenius-Euler polynomials arising from non-linear differential equations. J. Number Theory 132(12), 2854-2865 (2012)

12. Kim, T: An identity of the symmetry for the Frobenius-Euler polynomials associated with the fermionic $p$-adic invariant $q$-integrals on $\mathbb{Z}_{p}$. Rocky Mt. J. Math. 41, 239-247 (2011)

13. Rim, S-H, Joung, J, Jin, J-H, Lee, S-J: A note on the weighted Carlitz's type $q$-Euler numbers and $q$-Bernstein polynomials. Proc. Jangjeon Math. Soc. 15, 195-201 (2012)

14. Rim, S-H, Lee, S-J: Some identities on the twisted $(h, q)$-Genocci numbers and polynomials associated with q-Bernstein polynomials. Int. J. Math. Sci. 2011, Article ID 482840 (2011)

15. Roman, S: The Umbral Calculus. Dover, New York (2005)

16. Ryoo, CS, Agarwal, RP: Calculating zeros of the Frobenius-Euler polynomials. Neural Parallel Sci. Comput. 17, 351-361 (2009)

17. Simsek, Y, Bayad, A, Lokesha, V: $q$-Bernstein polynomials related to $q$-Frobenius-Euler polynomials, l-functions, and q-Stirling numbers. Math. Methods Appl. Sci. 35, 877-884 (2012)

18. Simsek, Y, Yurekli, O, Kurt, V: On interpolation functions of the twisted generalized Frobenius-Euler numbers. Adv. Stud. Contemp. Math. 15, 187-194 (2007)

19. Simsek, Y: Special functions related to Dedekind-type DC-sums and their applications. Russ. J. Math. Phys. 17, 495-508 (2010)

doi:10.1186/1687-1847-2012-222

Cite this article as: Kim et al.: Frobenius-Euler polynomials and umbral calculus in the $p$-adic case. Advances in Difference Equations 2012 2012:222.

\section{Submit your manuscript to a SpringerOpen ${ }^{\odot}$ journal and benefit from:}

- Convenient online submission

Rigorous peer review

- Immediate publication on acceptance

- Open access: articles freely available online

- High visibility within the field

- Retaining the copyright to your article 